\title{
Salt-inducible kinase 1 is involved in high glucose-induced mesangial cell proliferation mediated by the ALK5 signaling pathway
}

\author{
JIE YU ${ }^{1,2^{*}}$, XIAOPENG HU ${ }^{3 *}$, ZHENGDONG YANG $^{4}$, HIROSHI TAKEMORI $^{5}$, YANHONG LI ${ }^{1}$, \\ HUAN ZHENG $^{1}$, SHICONG HONG ${ }^{1}$, QIUHONG LIAO $^{1}$ and XIUYING WEN ${ }^{1}$ \\ ${ }^{1}$ Department of Traditional Chinese Medicine and Endocrinology, Liyuan Hospital, Tongji Medical College, \\ Huazhong University of Science and Technology, Wuhan; ${ }^{2}$ First People's Hospital of Jiujiang City, Jiujiang; \\ ${ }^{3}$ Department of General Surgery, Tongji Hospital and ${ }^{4}$ Department of Neurology, Liyuan Hospital, \\ Tongji Medical College, Huazhong University of Science and Technology, Wuhan, P.R. China; \\ ${ }^{5}$ Laboratory of Cell Signaling and Metabolism, National Institute of Biomedical Innovation, Osaka, Japan
}

Received February 16,2013; Accepted April 16, 2013

DOI: $10.3892 /$ ijmm.2013.1377

\begin{abstract}
High glucose levels can induce mesangial cell proliferation and extracellular matrix (ECM) accumulation through the type I activin receptor-like kinase 5 (ALK5) signaling pathway. Salt-inducible kinase 1 (SIK1) prevents fibrosis by downregulating ALK5, while the expression level of the SIK1 protein itself is downregulated by glucose in neuronal cells following ischemia. In this study, we investigated the correlation between SIK1 and the ALK5 signaling pathway in a rat glomerular mesangial cell line (HBZY-1 cells). We found that high glucose levels downregulated the expression level of SIK1 and suppressed the phosphorylation of SIK1 at Thr-182. The downregulation of SIK1 by high glucose was accompanied by the activation of the ALK5 signaling pathway, while the overexpression of SIK1 in the HBZY-1 cells resulted in a decrease in the ALK5 protein level, as well in the levels of its downstream targets, including fibronectin and plasminogen activator inhibitor type I. In conclusion, high glucose may activate the ALK5 signaling pathway by downregulating SIK1, and SIK1 may be a protective factor against cellular proliferation and ECM accumulation in glomerular mesangial cells under high glucose conditions.
\end{abstract}

Correspondence to: Dr Xiuying Wen, Department of Traditional Chinese Medicine and Endocrinology, Liyuan Hospital, Tongji Medical College, Huazhong University of Science and Technology, 39 Lake Avenue, Wuhan, Hubei 430077, P.R. China

E-mail: wenxyoung@hust.edu.cn

*Contributed equally

Key words: extracellular matrix accumulation, activin receptor-like kinase 5 signaling pathway, salt-inducible kinase 1, cell proliferation

\section{Introduction}

Diabetic nephropathy is one of the most prevalent causes of end-stage renal disease. Increasing evidence has indicated that diabetic nephropathy in experimental animals, as well as in diabetic patients, induces fibrotic changes, such as extracellular matrix (ECM) accumulation in glomerular mesangial cells, which manifest as overt nephropathy (1). In vitro studies have also revealed that high glucose levels induce the overt proliferation of mesangial cells and ECM accumulation through a complex pathological mechanism $(2,3)$. Transforming growth factor- $\beta$ (TGF- $\beta$ ) has been shown to play a central role in the progression of glomerular fibrosis and to transduce its signal through the type I activin receptor-like kinase 5 (ALK5) (4). Additionally, TGF- $\beta$ specifically induces the expression of the large modular glycoprotein, fibronectin $(\mathrm{FN})$, and the antifibrinolytic agent, plasminogen activator inhibitor type 1 (PAI-1), resulting in cellular proliferation and ECM accumulation (5). A number of studies have shown that the selective inhibition of ALK5 may be a potential therapeutic strategy for the treatment of chronic renal disease secondary to diabetic nephropathy $(6,7)$.

Salt-inducible kinase 1 (SIK1), a serine/threonine protein kinase, was originally found in the adrenal glands of rats fed a high salt diet (8). SIK1 is belongs to the family of 5' adenosine monophosphate (AMP)-activated protein kinases (AMPKs), since its amino acid sequence is closely related to the catalytic $\alpha$ subunit of AMPK $(8,9)$. Additionally, the AMPK-related kinases share some functional properties, several substrates and the upstream kinase, serine/threonine kinase 11 (STK11, also termed LKB1). SIK1 is predominantly localized in the nucleus in resting Y1 cells, where it supresses cAMP-response element binding protein (CREB)-mediated gene expression by phosphorylating the co-activator, CREB-regulated transcription co-activator (CRTC). Following treatment with adrenocorticotropic hormone (ACTH) and the subsequent phosphorylation of the regulatory domain at Ser- 577 by protein 
kinase A (PKA), SIK1 translocates to the cytoplasm and loses its repressive properties (10).

In contrast to the negative regulation by PKA, LKB1 phosphorylates SIK1 at Thr-182 in the activation loop (A-loop) of the kinase domain, which is essential for catalytic activity. The autophosphorylation at Ser-186, which is 4 amino acids downstream from Thr-182, is also important for the sustained activity of SIK1 $(11,12)$. Previous studies on CREB and LKB1 have revealed a number of physiological roles for SIK1, such as cell cycle regulation (13), muscle growth and differentiation (14) and tumor suppression (15). In diabetic states, SIK1 is subject to hormonal control and constrains gluconeogenic and lipogenic gene expression in the liver $(16,17)$.

Cell biology has also identified SIK1 as a target of the TGF- $\beta /$ Smad signaling pathway. SIK1 forms complexes with ALK5, Smad7, and Smurf2, which results in the downregulation of the TGF- $\beta$-mediated activation of the ALK5 signaling pathway. Following SIK1 knockdown by siRNA, endogenous ALK5 levels are significantly higher, and the decreased repression of TGF- $\beta$ signaling augments the induction of the expression of FN and PAI-1 $(18,19)$, both of which are involved in fibrotic disorders, such as glomerulosclerosis. These findings suggest that SIK1 may serve as a therapeutic target against fibrosis through the specific downregulation of ALK5.

Although animal experiments have found the highest SIK mRNA levels in the rat kidney (20), to our knowledge, there are no studies available concerning the exact role of SIK1, which is involved in the diabetic kidney. There is also limited knowledge concerning the specific role of SIK1 in glucose metabolism, cellular proliferation and ECM accumulation. In the current study, we report that the protein level of SIK1 is downregulated by high glucose in a rat glomerular mesangial cell line (HBZY-1 cells). By contrast, the overexpression of SIK1 in HBZY-1 cells results in the reduction of cell proliferation and ECM accumulation accompanied by the inhibition of the ALK5 signaling pathway.

\section{Materials and methods}

Reagents and chemicals. The PCR primers and Lipofectamine 2000 were purchased from Invitrogen (Carlsbad, CA, USA). The restriction enzymes, BamHI, EcoRI and T4 DNA ligase, were obtained from Takara Co. (Kyoto, Japan). Protein G Sepharose was purchased from GE Healthcare (Piscataway, NJ, USA). Rabbit anti-rat SIK1/PAI-1/FN polyclonal antibody was purchased from Santa Cruz Biotechnology, Inc. (Santa Cruz, CA, USA). Rabbit polyclonal antibody against phospho-SIK1 (phospho-Thr-182) and anti-SIK1 antiserum were a gift from Dr H. Takemori, Laboratory of Cell Signaling and Metabolism, National Institute of Biomedical Innovation, Osaka, Japan. Rabbit anti-rat ALK5 polyclonal antibody was purchased from Bioworld Technology, Inc. (St. Louis Park, MN, USA).

Expression plasmid construction. The expression plasmid, pCDF1-MCS2-EF1-copGFP, was purchased from System Biosciences (Mountain View, CA, USA). The plasmid vector of pEGFP and the E. coli strain, DH5 $\alpha$, were preserved in our laboratory. The full-length cDNA coding for rat SIK1 was amplifiedusing the following primers: 5'-GCGGATCCGGTGC GGCCCGAAGCCATGGTGATCATGTCGGAGTTCAG-3' and 5'-CCGGATCCATGGTGATCATGTCGGAGTTCAG-3' and was cloned into the $B a m \mathrm{HI} / E c o$ RI site of pCDF1-MCS2; the full-length rat SIK1 cDNA was subsequently subcloned into pEGFP-C1.

Cell culture and gene transfer. HBZY-1 cells, a rat glomerular mesangial cell line, were purchased from the China Center for Type Culture Collection (Wuhan, China), maintained at $37^{\circ} \mathrm{C}$ in a humidified atmosphere of $5 \% \mathrm{CO}_{2}$ and cultured in Dulbecco's modified Eagle's medium (DMEM; Gibco-BRL, Gaithersburg, MD,USA) supplemented with $10 \%$ fetal bovine serum, $100 \mathrm{U} / \mathrm{ml}$ penicillin and $100 \mu \mathrm{g} / \mathrm{ml}$ streptomycin. The cells were grown to $\sim 80 \%$ confluence and then incubated in serum-free medium for $24 \mathrm{~h}$. To investigate the effects of glucose on the protein expression of SIK1 and pT182-SIK1, serum-deprived HBZY-1 cells were cultured in DMEM containing $30 \mathrm{mM}$ glucose (high glucose) for $0,12,24$ and $48 \mathrm{~h}$. At the end of each time period, SIK1, pT182-SIK1, ALK5, FN and PAI-1 expression levels were examined. To determine the direct effects of high glucose on the specific cellular localization of SIK1, the HBZY-1 cells were transfected with the expression vectors for green fluorescence protein (GFP)-fused full-length SIK1 (pEGFP-SIK1), cultured with high glucose in glass-bottomed culture dishes (GBD-35-20; Nest Biotechnology Co., Ltd., Shanghai, China) for 0 and $48 \mathrm{~h}$, and then observed under an Olympus confocal microscope. To examine how SIK1 negatively regulates the ALK5 signaling pathway, HBZY-1 cells were randomly divided into three groups: a non-transfected normal group, an empty vector control group and a pCDF1-SIK1 group. At $48 \mathrm{~h}$ after transfection, the HBZY-1 cells were collected and analyzed for SIK1 expression. The cells were cultured with high glucose for a further $48 \mathrm{~h}$ to analyze SIK1, ALK5, FN and PAI-1 expression by western blot and immunocytochemistry analyses. The transient transfection of HBZY-1 cells was carried out using Lipofectamine 2000 according to the manufacturer's instructions. At $48 \mathrm{~h}$ after transfection, a fluorescent microscope was used to examine GFP expression, and the cells were then collected for protein extraction.

Immunocytochemistry and immunocytofluorescent analysis. Cells were cultured on glass coverslips in 12-well plates for 0 , 12,24 and $48 \mathrm{~h}$, gently washed twice with phosphate-buffered saline (PBS) and fixed with $4 \%$ polyformaldehyde for $20 \mathrm{~min}$ at room temperature. The cells were then washed three times with PBS, and the coverslips with the cells were made permeable with $0.3 \%$ Triton X-100 in PBS for 15 min. The cells were then blocked by pre-treatment with $3 \% \mathrm{H}_{2} \mathrm{O}_{2}$ for $10 \mathrm{~min}$ at $37^{\circ} \mathrm{C}$. Finally, the HBZY-1 cells were incubated with rabbit antiPAI-1/FN polyclonal antibody (1:100) overnight at $4^{\circ} \mathrm{C}$. The following day, the cells were washed with PBS three times and incubated with polymer helper for $30 \mathrm{~min}$ at $37^{\circ} \mathrm{C}$. Thereafter, the HBZY-1 cells were washed three times with PBS and then treated for $30 \mathrm{~min}$ at $37^{\circ} \mathrm{C}$ with polyperoxidase-anti-rabbit IgG. After being washed with PBS, the coverslips were stained with DAB. Negative controls were obtained by replacing specific antibody with non-immune serum. Images were taken using a light Olympus microscope. For indirect immunocytofluorescence, HBZY-1 cells on glass coverslips were fixed with $4 \%$ polyformaldehyde in $0.1 \%$ Triton X-100 and blocked in PBS containing 5\% goat serum. An antibody against SIK1 
was used at a dilution of 1:100. AlexaFluor 488-conjugated secondary antibodies were also used at a dilution of 1:200. Counterstaining of the nuclei was performed by the addition of $1 \mathrm{mM}$ 4',6-diamidino-2-phenylindole (DAPI) (Boster Biological Technology Ltd., Wuhan, Hubei, China) for $15 \mathrm{~min}$. Images were taken using an Olympus confocal microscope.

Reverse transcription-polymerase chain reaction (RT-PCR). The cells were washed twice with PBS. RT-PCR was performed as previously described (21). The primers used were: SIK1 sense, 5'-AGCACCACTCAGCCGTCTCATCT-3' and antisense, 5'-CAGGTCCTCCATCTCACAATCCC-3'; and $\beta$-actin sense, 5'-CGTTGACATCCGTAAAGACCTC-3' and antisense, 5'-TAGGAGCCAGGGCAGTAATCT-3'. After electrophoresis the gels were recorded using a digital recorder, and the mRNA expression levels were semi-quantified using ImageJ software (National Institutes of Health, USA).

Western blot analysis and immunoprecipitation. Western blot analysis was carried out as previously described (22). For immunoprecipitation, the proteins (500 $\mu \mathrm{g} /$ sample) extracted from the cells were incubated with anti-SIK1 antiserum for $16 \mathrm{~h}$ at $4^{\circ} \mathrm{C}$ with rotation. Protein $\mathrm{G}$ agarose beads ( $\left.20 \mu \mathrm{l} / \mathrm{sample}\right)$ were then added to each sample and incubated for an additional $2 \mathrm{~h}$ at $4^{\circ} \mathrm{C}$ with rotation. The complexes were spun down, and the pellet was washed three times with immunoprecipitation buffer. The immunoprecipitates were immobilized on a polyvinylidene difluoride membrane after separation on $12 \%$ SDS-PAGE gels. The membrane was immunoblotted with antipT182-SIK1 antibody by enhanced chemiluminescence (ECL) (Pierce Chemical, Co., Rockford, IL, USA) method. The same membrane was subsequently reblotted with anti-SIK1 antibody after stripping to verify that equivalent amounts of SIK1 protein were loaded onto each lane, and the protein expression levels were semi-quantified using ImageJ software.

Cell proliferation measurements. Cell proliferation was measured using a dimethylthiazol-diphenyltetrazolium bromide (MTT) assay in 96-well microplates. HBZY-1 cells were exposed to high glucose for different periods of time with or without gene transfection. Medium $(180 \mu \mathrm{l})$ was added to each well. After $48 \mathrm{~h}, 20 \mu \mathrm{l}$ of $5 \mathrm{mg} / \mathrm{ml}$ MTT were added to each well. Four hours later, the medium was replaced with $200 \mu \mathrm{l}$ of dimethyl sulfoxide, and the 96-well microplate was shaken gently. The absorbance was then measured at $570 \mathrm{~nm}$, and these data were transformed into a variable representing the number of cells in each well using a standard curve that correlated the absorbance to the number of HBZY-1 cells.

Statistical analysis. Data analysis was conducted using GraphPad Prism 5.0 software, and the data are expressed as the means \pm SEM. Comparisons among multiple groups were performed using one-way ANOVA followed by a Newman-Keuls test. A P-value $<0.05$ was considered to indicate a statistically significant difference.

\section{Results}

High glucose levels suppress the activity and decrease the expression of SIKI and regulate the specific localization and distribution of SIK1 in HBZY-1 cells. HBZY-1 cells were stimulated with high glucose, and the activity resulting from the Thr-182 phosphorylation of the SIK1 gene was monitored. The HBZY-1 cells were cultured in medium containing $30 \mathrm{mM}$ glucose for $0,12,24$, or $48 \mathrm{~h}$. Immunoprecipitation followed by western blot analysis revealed that SIK1 activity decreased following exposure with glucose in a time-dependent manner (Fig. 1A). The levels of Thr-182 phosphorylation at 12, 24 and $48 \mathrm{~h}$ were $24.8,58$ and $58.4 \%$, respectively, compared to $0 \mathrm{~h}$. The level of phospho-Thr-182 is important to maintain the SIK1 protein level (12), which was also evident in the HBZY-1 cells treated with high glucose (Fig. 1B). The density ratios of SIK1/ $\beta$-actin at 12, 24 and $48 \mathrm{~h}$ were 18.2,61.2 and 72\%, respectively, compared to $0 \mathrm{~h}$. Further semi-quantitative RT-PCR analysis also demonstrated equivalent results at the transcriptional level under high glucose conditions at each time point (Fig. 1C). These results demonstrate that high glucose concentrations suppress the activity and decrease the expression of SIK1.

Since the intracellular distribution of SIK1 reflects its functional activity (19), we decided to examine the localization of SIK1 protein in the HBZY-1 cells. As shown in Fig. 1D, immuno-positive signals for SIK1 were detected in the cytoplasm and nucleus of the unstimulated HBZY-1 cells. Followign stimulation with high glucose, the SIK1 signals were gradually detected in the nucleus with decreased intensity.

To confirm our results from immunocytochemical analysis, green fluorescence protein (GFP)-tagged SIK1 protein was transfected into the HBZY-1 cells and the subcellular translocation of GFP signals was monitored before and after exposure to high glucose. In the unstimulated cells, the GFP-SIK1 signal was observed in both the nuclear and cytoplasmic compartments (Fig. 1E). When the cells were treated with high glucose, the green fluorescence signal was subsequently translocated into the nucleus from the cytoplasm. When the GFP-tag alone was introduced into the HBZY-1 cells, the fluorescence signal was not localized at any precise subcellular area, and its distribution did not change after exposure to high glucose (data not shown). These results indicate that high glucose levels induce the nuclear import of SIK1, which may result from the reduction in SIK1 kinase activity.

High glucose induces the activation of the ALK5 signaling pathway in HBZY-1 cells. The ALK5 signaling pathway has been shown to be induced by high glucose levels and promotes cell proliferation with ECM accumulation $(23,24)$. Western blot analysis using HBZY-1 cells also showed that the level of ALK5 was upregulated by high glucose levels (Fig. 2A) with 1.82- and 2.92-fold inductions at 24 and $48 \mathrm{~h}$, respectively (normalized with $\beta$-actin), whereas no significant difference in the ALK5 level was observed between 0 and $12 \mathrm{~h}$. In contrast to ALK5, the protein levels of FN and PAI-1, downstream targets of ALK5, were also increased by high glucose levels in a time-dependent manner. Immunocytochemical analysis of $\mathrm{FN}$ (Fig. 2B) and PAI-1 (Fig. 2C) protein in the HBZY-1 cells also provided consistent results at $48 \mathrm{~h}$, suggesting that high glucose levels induce the activation of the ALK5 signaling pathway, which may be attributed to the downregulation of SIK1.

Forced expression of SIK1 induces the degradation of ALK5 and reduces ECM production in HBZY-1 cells. To determine 
A
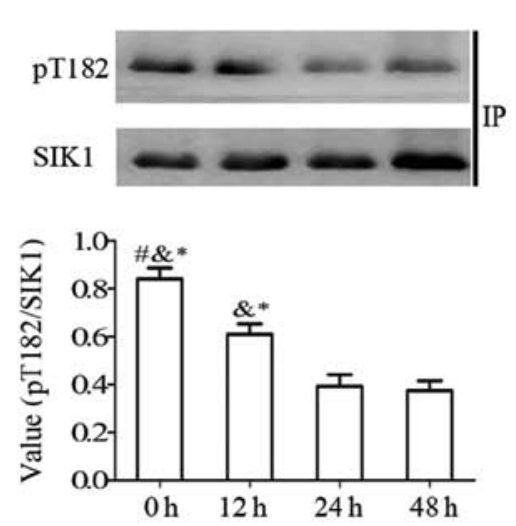

B
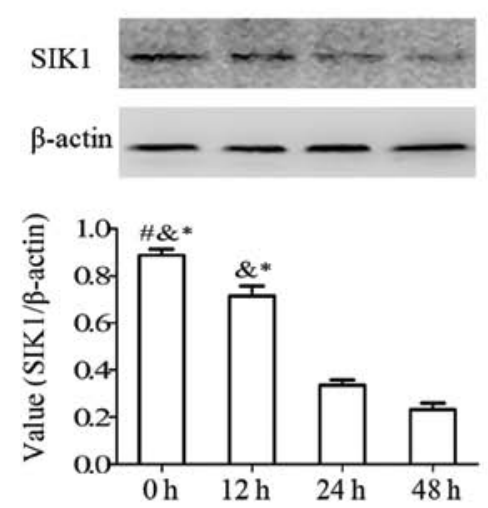

$\mathbf{C}$
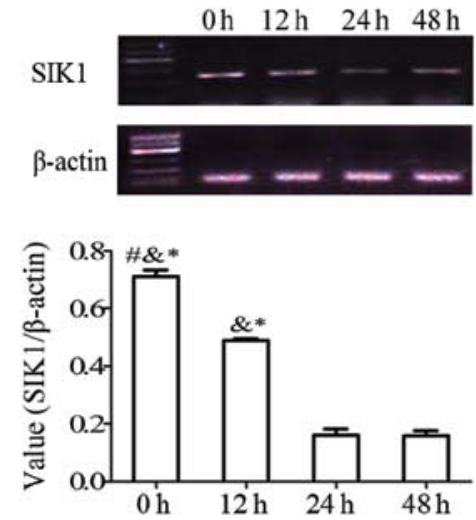
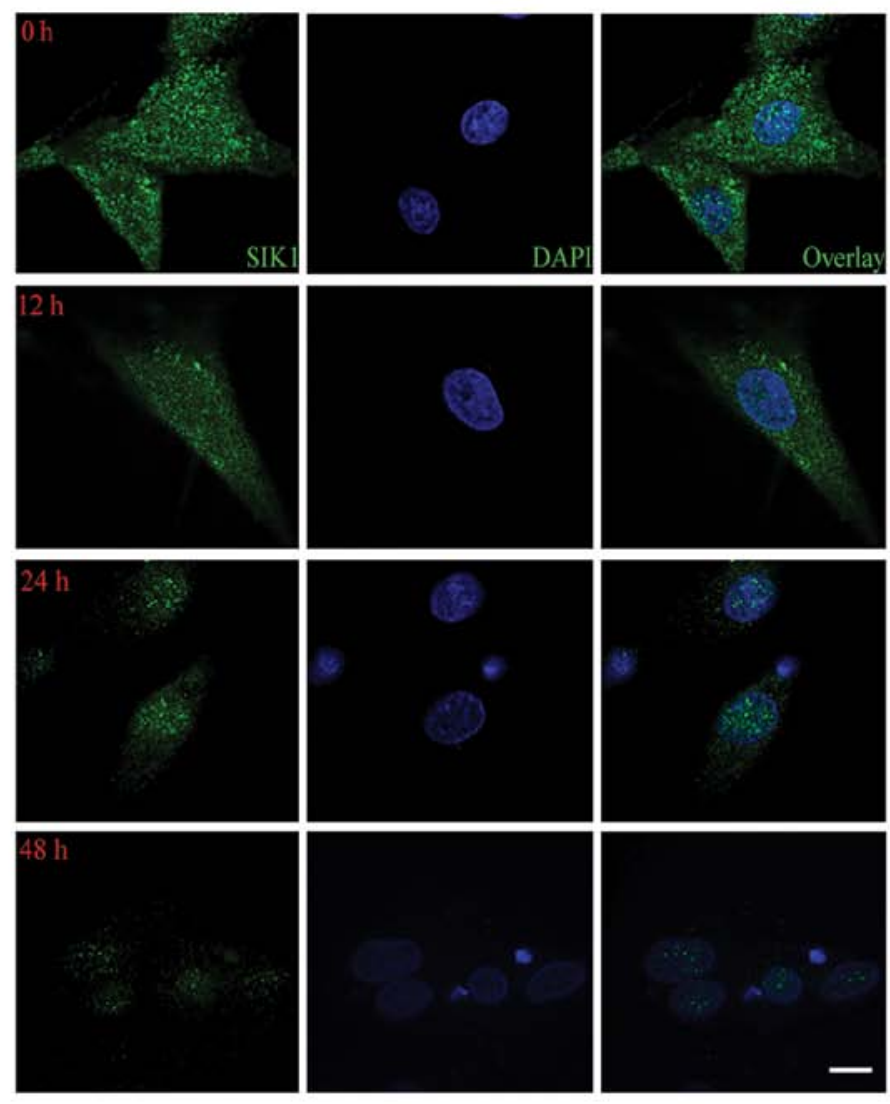

$\mathbf{E}$
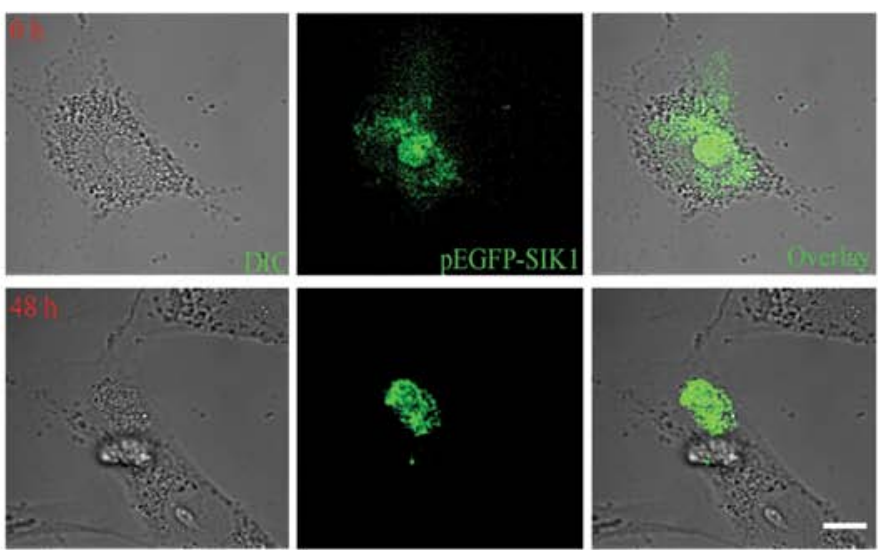

Figure 1. High glucose levels significantly suppress the expression and activity of salt-inducible kinase 1 (SIK1) and regulate the nuclear redistribution of SIK1 in HBZY-1 cells. (A) Immunoprecipitation and western blot analysis showing the levels of pT182-SIK1 and the SIK1 control in HBZY-1 cells cultured in high glucose $(30 \mathrm{mM})$ at $0,12,24$, and $48 \mathrm{~h}$. (B) Western blot analysis showing the levels of SIK1 protein and the $\beta$-actin control at different culture times. (C) RT-PCR showing the levels of SIK1 mRNA and the $\beta$-actin control in HBZY-1 cells at different culture times. (D) Representative confocal images of HBZY-1 cells that were cultured on coverslips in high glucose for 0,12, 24, $48 \mathrm{~h}$ for immunocytofluorescent analysis. Green fluorescent signals of endogenous SIK1 (left panel), blue fluorescent signals representing nuclear staining with DAPI (middle panel), and merged images of both (right panel). Scale bar, $10 \mu \mathrm{m}$. (E) Representative confocal images of HBZY-1 cells cultured in glass-bottomed culture dishes and transfected with overexpression vectors for green fluorescence protein (GFP)-fused full-length SIK1 (pEGFP-SIK1) protein stimulated with high glucose for 0 and $48 \mathrm{~h}$. Confocal DIC images of HBZY-1 cells (left panel), green fluorescent signals of GFP-SIK1 (middle panel), and overlaid images of both (right panel). Scale bar, $10 \mu \mathrm{m} .{ }^{\#} \mathrm{P}<0.05 \mathrm{vs}$. at time of $12 \mathrm{~h} ;{ }^{\circledR} \mathrm{P}<0.05 \mathrm{vs}$. at time of $24 \mathrm{~h}$; "P<0.05 vs. at time of $48 \mathrm{~h}$.

the involvement of the reduced levels of SIK1 in the upregulation of ALK5 signaling in glomerular mesangial cells, we performed paradoxical assays through the enforced expression of SIK1 in HBZY-1 cells. The transformation efficiency, monitored by the control copGFP-expression, was $\sim 60 \%$ (data not shown). When the SIK1 expression plasmid was transformed into the HBZY-1 cells, the SIK1 protein (Fig. 3A) and mRNA
(Fig. 3B) levels increased by $\sim 1.8$ - and 1.9-fold, respectively, compared to the non- or mock-transfected cells. Following the stimulation of the cells with high glucose for $48 \mathrm{~h}$, a negative correlation between the levels of SIK1 protein and ALK5, FN and PAI-1 protein leels was observed (Fig. 3C). When the SIK1 expression plasmid was transformed into the HBZY-1 cells, the ALK5 protein expression decreased by $\sim 38 \%$ in the 

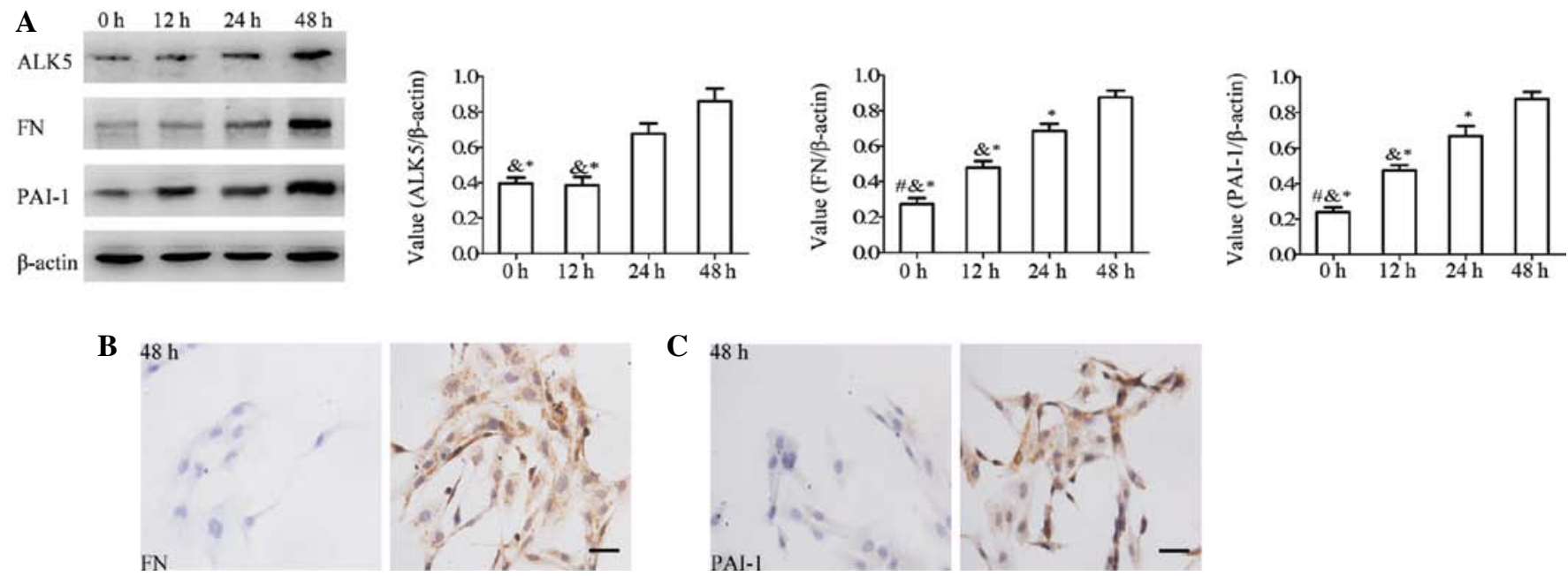

Figure 2. High glucose levels significantly induce the activation of the activin receptor-like kinase 5 (ALK5) signaling pathway in HBZY-1 cells. (A) Western blot analysis showing the levels of ALK5, fibronectin (FN), plasminogen activator inhibitor type 1 (PAI-1) protein and the $\beta$-actin control at different culture times. (B) Representative microscopic images of HBZY-1 cells that were cultured on coverslips in high glucose for 0 and $48 \mathrm{~h}$ for immunocytochemical analysis of FN protein. Scale bar, $50 \mu \mathrm{m}$. (C) Representative microscopic images of HBZY-1 cells for immunocytochemical analysis of PAI-1 protein in high glucose at 0 and $48 \mathrm{~h}$. Scale bar, $50 \mu \mathrm{m} .{ }^{\#} \mathrm{P}<0.05$ vs. at time $12 \mathrm{~h} ;{ }^{\circledR} \mathrm{P}<0.05 \mathrm{vs}$. at time of $24 \mathrm{~h} ;{ }^{*} \mathrm{P}<0.05 \mathrm{vs}$. at time of $48 \mathrm{~h}$.

A

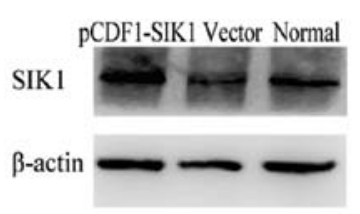

C

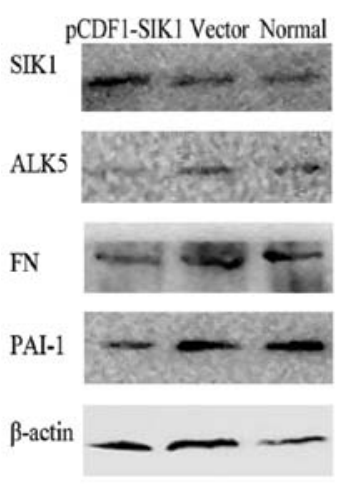

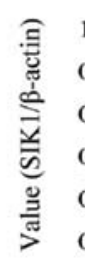
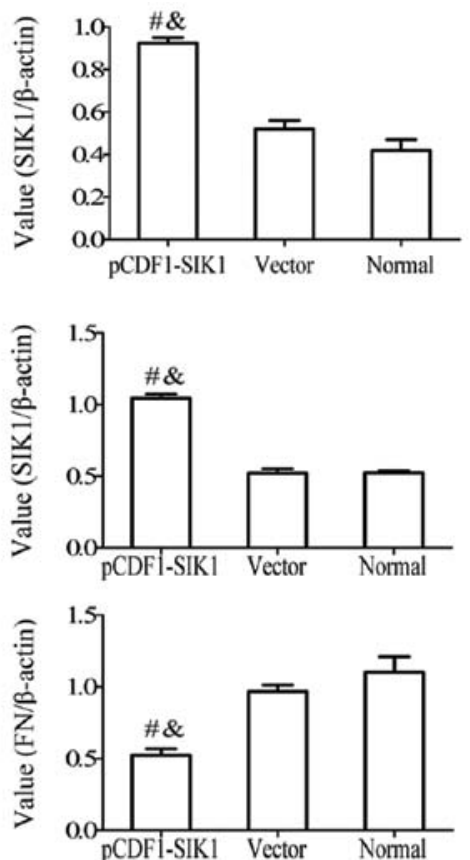

B

SIK 1 pCDFI-SIKI Vector Normal

$\beta$-actin
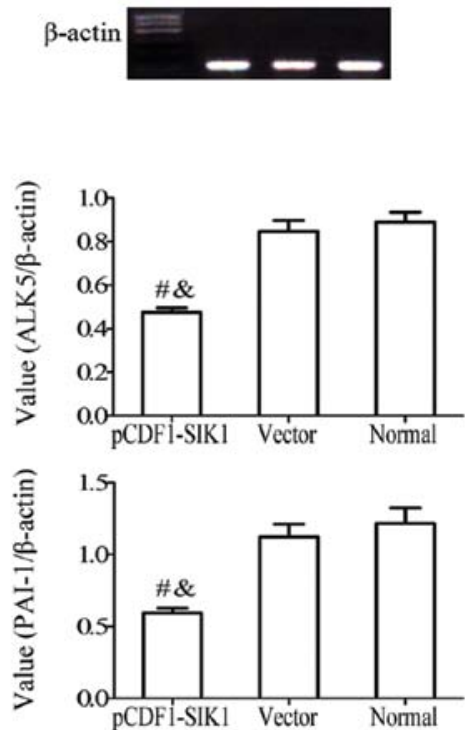

D

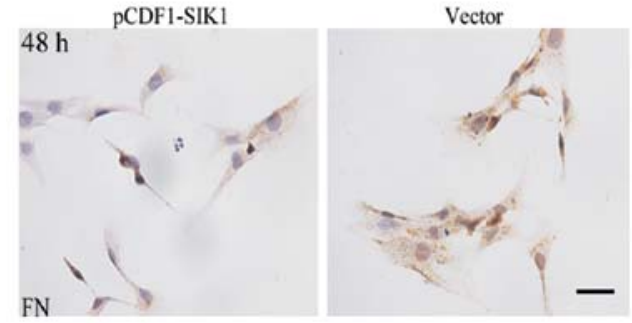

E

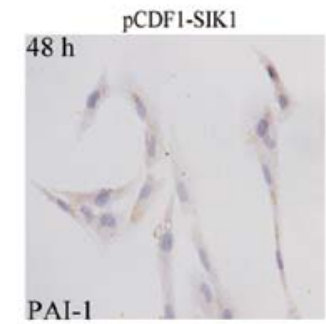

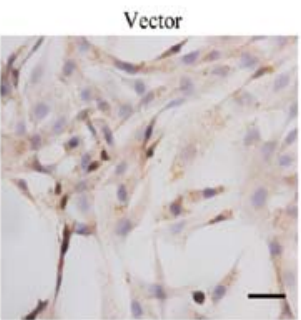

Figure 3. Overexpression of salt-inducible kinase 1 (SIK1) in HBZY-1 cells resulted in the downregulation of activin receptor-like kinase 5 (ALK5) and its target gene, fibronectin (FN) and plasminogen activator inhibitor type 1 (PAI-1). (A) Western blot analysis showing increased levels of SIK1 protein from HBZY-1 cells transfected with SIK1 expression vector (pCDF1-SIK1) or control vector. Protein levels were normalized to the respective $\beta$-actin control. (B) RT-PCR showing increased levels of SIK1 mRNA in HBZY-1 cells following transfection. The mRNA levels were normalized to the respective $\beta$-actin control. (C) Western blot analysis showing increased levels of SIK1 and decreased ALK5, FN and PAI-1 protein levels in HBZY-1 cells cultured with high glucose for 48 h after transfection. Protein levels were normalized to the respective $\beta$-actin control. (D) Representative microscopic images of HBZY-1 cells which were cultured on coverslips in high glucose for $48 \mathrm{~h}$ after transfection for immunocytochemical analysis of FN protein. Scale bar, $50 \mu \mathrm{m}$. (E) Representative microscopic images of HBZY-1 cells after transfection for immunocytochemical analysis of PAI-1 protein. Scale bar, $50 \mu \mathrm{m}$. ${ }^{\sharp} \mathrm{P}<0.05 \mathrm{vs}$. empty vector control group at $48 \mathrm{~h}$; ${ }^{\&} \mathrm{P}<0.05$ vs. normal control group at $48 \mathrm{~h}$. 


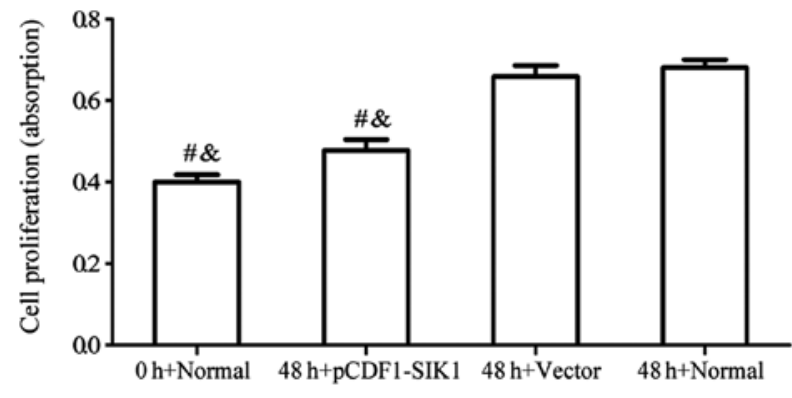

Figure 4. Overexpression of salt-inducible kinase 1 (SIK1) in HBZY-1 cells significantly reverses the high glucose-induced HBZY-1 cell overproliferation. MTT assays showing the absorbance values for HBZY-1 cells before transfection at 0 and $48 \mathrm{~h}$ and after transfection at $48 \mathrm{~h} .{ }^{*} \mathrm{P}<0.05 \mathrm{vs}$. empty vector control group at $48 \mathrm{~h} ;{ }^{\&} \mathrm{P}<0.05$ vs. normal control group at $48 \mathrm{~h}$.

pCDF1-SIK1 transfected cells compared to the control cells. Immunocytochemical analysis of FN (Fig. 3D) and PAI-I (Fig. 3E) also supported the negative correlation between SIK1 and ALK5 signaling.

Upregulation of SIK1 reverses the high glucose-induced overproliferation of HBZY-1 cells. To determine whether SIK1 exerts antiproliferative effects on HBZY-1 cells, we analyzed HBZY-1 cell proliferation using MTT assays. The HBZY-1 cells were transfected with or without pCDF1-SIK1 and subjected to high glucose levels for 48 h. As shown in Fig. 4, a significant reduction in the proliferation of HBZY-1 cells due to the overexpression of SIK1 was observed.

\section{Discussion}

The progression of diabetic nephropathy to end-stage kidney disease is manifested by the gradual, inexorable process of renal glomerular fibrosis. Several studies have attempted to develop effective therapies and preventive strategies for diabetic nephropathy by elucidating the molecular mechanisms that lead to chronic glomerular fibrosis. SIK1 may be a therapeutic target for diabetic nephropathy as it is a negative regulator of the ALK5 signaling pathway and is abundantly expressed in the rat kidney.

In the present study, we demonstrate that high glucose levels suppress SIK1 activity in HBZY-1 cells. This result is consistent with evidence showing that AMPK-related kinases, such as AMPK, SIK2 (an isoform of SIK1) and SNF1/AMPK-related kinase (SNARK), are activated by glucose deprivation (25). A proposed mechanism for high glucose-induced SIK1 suppression could be traceable to its upstream kinase, LKB1. Suppressed LKB1 signaling by high glucose may contribute to the decreased activity of SIK1 $(11,26)$. Similar to the kinase activity levels of SIK1, the intracellular distribution of SIK1 has also been shown to be a marker of the LKB1-mediated regulation of SIK1 activity.

Since Ser-577, a determinant of the intracellular distribution of SIK1, is also an autophosphorylation site, inactive SIK1 as well as CREB-repressing active SIK1 are present as dephosphoforms at Ser-577 and are localized in the nucleus (27). Therefore, SIK1 is localized only in the nucleus in LKB1-defective HeLa cells, and LKB1 overexpression isolates a portion of SIK1 into the cytoplasm, whereas the combination of LKB1 and PKA completely induces the cytoplasmic localization of SIK1 (28).

In this study, indirect immunocytofluorescence and GFP-tagged SIK1 revealed the nuclear redistribution of SIK1 following stimulation with high glucose, suggesting that high glucose levels decreased the activity of SIK1, most likely inactivating the upstream kinase, LKB1. The correlation of the decreased activity of SIK1 with its protein level in HBZY-1 cells under high glucose conditions is also consistent with findings in HeLa cells in which the level of phospho-Thr-182/ Ser-186 correlated with the SIK1 protein level (11). By contrast, the elevated levels of SIK1 mRNA and protein in Y1 cells have been shown to be accompanied by an increase in SIK1 kinase activity (27). This suggests that the reduction in SIK1 protein levels in HBZY-1 cells following exposure to high glucose may result from inactive SIK1 and decreased SIK1 mRNA levels.

Glucose induces $\mathrm{Ca}^{2+}$ signaling, which activates $\mathrm{Ca}^{2+}$ calmodulin-dependent kinases (CaMKs). CaMK I/IV phosphorylates SIK2 at Thr-484 in the motif of RRHT (29), which is conserved in SIK1. Subsequently, phospho-SIK2 is degraded by the proteasome, which restores the signaling cascades that have been suppressed by SIK2, such as CREB-TORC1. The fact that glucose toxicity induces the activation of proteasomes (30) and CaMK I/IV (31), along with the findings that high glucose levels induce TGF- $\beta$ /ALK 5 signaling followed by cell proliferation and ECM accumulation in mesangial cells $(4,32)$, suggests that glucose-mediated signaling pathways may suppress SIK1 function. This suppression may result in the activation of the ALK5 signaling pathway. However, there is a small discrepancy between the induction of ALK5 and the induction of its downstream targets. High glucose levels gradually upregulate ALK5 expression but rapidly induce FN and PAI-I expression, suggesting that an alternate pathway that is completely independent of ALK5 may induce ALK5 downstream. The delayed induction of ALK5 is consistent with previous studies showing that high glucose levels do not induce rapid changes in ALK5 mRNA or protein levels $(23,32)$, but that ALK5 is markedly induced after prolonged stimulation under high glucose conditions (23).

TGF- $\beta$ plays a central role in the progression of diabetic nephropathy. However, TGF- $\beta$ is a pleiotropic factor involved in a variety of biological processes, including modulation of cell growth, angiogenesis, ECM deposition and various immune responses (33). Many of these actions may be important to balance the response to chronic kidney injury. However, the appropriate levels and long-term effect of TGF- $\beta$ inhibition has always been controversial (34). From the many studies on the TGF- $\beta$ signaling pathway, it is becoming clear that ALK5 mediates many of the actions of TGF- $\beta$. Therefore, the direct inhibition of ALK5 represents an alternative and attractive mechanism for the prevention of the detrimental profibrotic effects of TGF- $\beta$. In this study, in vitro experimental data clearly demonstrated that high glucose levels activated the ALK5 signaling pathway and downregulated SIK1. In other words, upregulating SIK1 may be a beneficial method to reduce high glucose-induced proliferation and fibrosis by enhancing ALK5 degradation. To support this hypothesis, a specific plasmid for SIK1 was transfected into HBZY-1 cells using Lipofectamine 2000. As a result, there was a marked downregulation in ALK5 protein expression, resulting in the inhibition of 
FN and PAI-1 expression and cellular proliferation in addition to the antagonism of fibrosis processing mechanisms. Taken together, these findings indicate that SIK1 protects mesangial cells against high glucose-induced cellular proliferation and ECM accumulation through the inhibition of the activation of the ALK5 signaling pathway.

In conclusion, our results indicate that high glucose levels decrease SIK1 expression and activity in glomerular mesangial cells. The decrease in SIK1 expression reduces the pathogenesis of mesangial cell proliferation and ECM accumulation through the activation of the ALK5 signaling pathway. Given the importance of glucose in the pathogenesis of mesangial cell proliferation, the SIK1-mediated regulation of ALK5 may provide innovative strategies for the treatment of diseases involving mesangial cells, such as glomerular fibrosis.

\section{Acknowledgements}

This study was supported by the Ministry of Education Specialized Research Fund for the Doctoral Program of Education (no. 20110142110016) and the Innovation Fund of Huazhong University of Science and Technology Ph.D. Dissertation (2011-2012).

\section{References}

1. Border WA and Noble NA: Evidence that TGF- $\beta$ should be a therapeutic target in diabetic nephropathy. Kidney Int 54: 1390-1391, 1998.

2. Ayo SH, Radnik RA, Glass W, et al: Increased extracellular matrix synthesis and mRNA in mesangial cells grown in high-glucose medium. Am J Physiol 260: F185-F191, 1991.

3. Paradis V, Perlemuter G, Bonvoust F, et al: High glucose and hyperinsulinemia stimulate connective tissue growth factor expression: a potential mechanism involved in progression to fibrosis in nonalcoholic steatohepatitis. Hepatology 34: 738-744, 2001.

4. Schnaper HW, Hayashida T, Hubchak SC and Poncelet AC: TGF- $\beta$ signal transduction and mesangial cell fibrogenesis. Am J Physiol Renal Physiol. 284: F243-F252, 2003.

5. Lebrin F, Deckers M, Bertolino P and Ten Dijke P: TGF- $\beta$ receptor function in the endothelium. Cardiovasc Res 65: 599-608, 2005.

6. Moon J, Kim H, Cho I, Sheen Y and Kim D: IN-1130, a novel transforming growth factor- $\beta$ type I receptor kinase (ALK5) inhibitor, suppresses renal fibrosis in obstructive nephropathy. Kidney Int 70: 1234-1243, 2006.

7. Laping NJ: ALK5 inhibition in renal disease. Curr Opin Pharmacol 3: 204-208, 2003

8. Wang Z, Takemori H, Halder SK, Nonaka Y and Okamoto M Cloning of a novel kinase (SIK) of the SNF1/AMPK family from high salt diet-treated rat adrenal. FEBS Lett 453: 135-139, 1999.

9. Steinberg GR and Kemp BE: AMPK in health and disease. Physiol Rev 89: 1025-1078, 2009.

10. Takemori H, Katoh Y, Horike N, Doi J and Okamoto M: ACTH-induced nucleocytoplasmic translocation of salt-inducible kinase. J Biol Chem 277: 42334-42343, 2002.

11. Hashimoto YK, Satoh T, Okamoto $M$ and Takemori $H$ : Importance of autophosphorylation at Ser186 in the A-loop of salt inducible kinase 1 for its sustained kinase activity. J Cell Biochem 104: 1724-1739, 2008.

12. Al-Hakim AK, Goransson O, Deak M, et al: 14-3-3 cooperates with LKB1 to regulate the activity and localization of QSK and SIK. J Cell Sci 118: 5661-5673, 2005.

13. Romito A, Lonardo E, Roma G, Minchiotti G, Ballabio A and Cobellis G: Lack of sik1 in mouse embryonic stem cells impairs cardiomyogenesis by down-regulating the cyclin-dependent kinase inhibitor p57kip2. PloS One 5: e9029, 2010.
14. Berdeaux R, Goebel N, Banaszynski L, et al: SIK1 is a class II HDAC kinase that promotes survival of skeletal myocytes. Nat Med 13: 597-603, 2007.

15. Cheng H, Liu P, Wang ZC, et al: SIK1 couples LKB1 to p53-dependent anoikis and suppresses metastasis. Sci Signal 2: ra35, 2009.

16. Koo SH, Flechner L, Qi L, et al: The CREB coactivator TORC2 is a key regulator of fasting glucose metabolism. Nature 437: 1109-1111, 2005.

17. Yoon YS, Seo WY, Lee MW, Kim ST and Koo SH: Salt-inducible kinase regulates hepatic lipogenesis by controlling SREBP-1c phosphorylation. J Biol Chem 284: 10446-10452, 2009.

18. Kowanetz M, Lönn P, Vanlandewijck M, Kowanetz K, Heldin $\mathrm{CH}$ and Moustakas A: TGF $\beta$ induces SIK to negatively regulate type I receptor kinase signaling. J Cell Biol 182: 655-662, 2008

19. Lönn P, Vanlandewijck M, Raja E, et al: Transcriptional induction of salt-inducible kinase 1 by transforming growth factor $\beta$ leads to negative regulation of type I receptor signaling in cooperation with the Smurf2 ubiquitin ligase. J Biol Chem 287: 12867-12878, 2012.

20. Feldman JD, Vician L, Crispino M, Hoe W, Baudry M and Herschman HR: The Salt-inducible kinase, SIK, is induced by depolarization in brain. J Neurochem 74: 2227-2238, 2002.

21. Wen X, Zeng Y, Liu L, et al: Zhenqing recipe alleviates diabetic nephropathy in experimental type 2 diabetic rats through suppression of SREBP-1c. J Ethnopharmacol 142: 144-150, 2012.

22. Huang W, Yu J, Jia X, Xiong L, Li N and Wen X: Zhenqing recipe improves glucose metabolism and insulin sensitivity by repressing hepatic FOXO1 in type 2 diabetic rats. Am J Chin Med 40: 721-733, 2012.

23. Wang $\mathrm{S}$ and Hirschberg R: Diabetes-relevant regulation of cultured blood outgrowth endothelial cells. Microvasc Res 78: 174-179, 2009.

24. Hills CE, Al-Rasheed N, Willars GB and Brunskill NJ: C-peptide reverses TGF- $\beta 1$-induced changes in renal proximal tubular cells: implications for treatment of diabetic nephropathy. Am J Physiol Renal Physiol 296: F614-F621, 2009.

25. Du J, Chen Q, Takemori $\mathrm{H}$ and Xu H: SIK2 can be activated by deprivation of nutrition and it inhibits expression of lipogenic genes in adipocytes. Obesity (Silver Spring) 16: 531-538, 2008.

26. Lee MJ, Feliers D, Sataranatarajan K, et al: Resveratrol ameliorates high glucose-induced protein synthesis in glomerular epithelial cells. Cell Signal 22: 65-70, 2010.

27. Lin X, Takemori H, Katoh Y, et al: Salt-inducible kinase is involved in the ACTH/cAMP-dependent protein kinase signaling in Y1 mouse adrenocortical tumor cells. Mol Endocrinol 15: 1264-1276, 2001

28. Katoh Y, Takemori H, Lin X, et al: Silencing the constitutive active transcription factor CREB by the LKB1-SIK signaling cascade. FEBS J 273: 2730-2748, 2006.

29. Sasaki T, Takemori H, Yagita Y, et al: SIK2 is a key regulator for neuronal survival after ischemia via TORC1-CREB. Neuron 69: 106-119, 2011.

30. Xu J, Wu Y, Song $\mathrm{P}$, Zhang $\mathrm{M}$, Wang $\mathrm{S}$ and Zou $\mathrm{MH}$ : Proteasome-dependent degradation of guanosine 5'-triphosphate cyclohydrolase I causes tetrahydrobiopterin deficiency in diabetes mellitus. Circulation 116: 944-953, 2007.

31. Trumper A, Trumper K and Horsch D: Mechanisms of mitogenic and anti-apoptotic signaling by glucose-dependent insulinotropic polypeptide in beta (INS-1)-cells. J Endocrinol 174: 233-246, 2002.

32. Wu L and Derynck R: Essential role of TGF- $\beta$ signaling in glucose-induced cell hypertrophy. Dev Cell 17: 35-48, 2009.

33. Gouville AC, Boullay V, Krysa G, et al: Inhibition of TGF- $\beta$ signaling by an ALK5 inhibitor protects rats from dimethylnitrosamine-induced liver fibrosis. Br J Pharmacol 145: 166-177, 2009.

34. Yata Y, Gotwals P, Koteliansky V and Rockey DC: Dosedependent inhibition of hepatic fibrosis in mice by a TGF- $\beta$ soluble receptor: implications for antifibrotic therapy. Hepatology 35: 1022-1030, 2002. 